\title{
Fuzzy Rule Based Load Frequency Control in a Parallel AC - DC Interconnected Power Systems through HVDC Link
}

\author{
S.Ramesh \\ Research Scholar, Anna University - \\ Zone 6, Salem (TN), India.
}

\author{
A.Krishnan \\ Dean, K.S.R. College of Engineering, \\ Tiruchengode (TN), India.
}

\begin{abstract}
:
In this paper, a fuzzy logic controller is proposed for an application of HVDC link to stabilize the frequency oscillation in a parallel $\mathrm{AC}-\mathrm{DC}$ interconnected power systems. When an interconnected AC power system is subjected to a load disturbance, system frequency may be considerably disturbed and becomes oscillatory. By utilizing the system interconnections as the control channels of HVDC link, the tie line power modulation of HVDC link through interconnections is applicable for stabilizing the frequency oscillation of $\mathrm{AC}$ system. The conventional Integral controller does not yield adequate control performance. To overcome this problem Fuzzy Logic Controller (FLC) is employed with a set of control rules. The proposed control technique is studied for a two area non reheat thermal power systems. From simulation results, the performance of the FLC is better during load disturbances.
\end{abstract}

\section{KEYWORDS:}

AC - DC Interconnected System, High Voltage Direct Current (HVDC) Link, Area Control Error, Load Frequency Control, Fuzzy Logic Control,

\section{INTRODUCTION}

Power engineers have the responsibility to deliver economical, adequate and quality power to consumers. In order to achieve this, the power system must be maintained at the desired operating level by implementing modern control strategies. The control of power systems is becoming increasingly more complex due to large interconnections. In an interconnected network, a disturbance in one line, leads to effects on the neighbouring systems change in tie-line power and frequency, causing serious problem of Load Frequency Control (FLC). LFC is a simple mechanism to maintain or restore the frequency and tie - line power flow among the interconnected power systems within the specified limit $[1,2]$.

LFC is a very important factor in power system operation and control for supplying sufficient and reliable electric power with good quality. There has been continuing interest in designing load frequency controller with better performance during the past 30 years. Many control strategy for LFC have been proposed since the 1970s [3]. Recently, applications of power electronics devices in AC power systems provide attractive benefits of economics and innovative technologies. In particular, High-Voltage Direct Current transmission link (HVDC link) offers major advantages in meeting these requirements, including long distance overhead bulk power transmission, transmission between unsynchronized AC systems [4-6], and marine cable transmission.

A sophisticated advantage of HVDC link is the enhanced damping of $\mathrm{AC}$ transmission using power modulation via an HVDC link in a parallel AC-DC interconnected power system [4]. When an AC power system is subjected to load disturbance, the system frequency may be considerably perturbed from the operating frequency. This may cause severe problems in system frequency oscillations. The deviation of frequency oscillations, that exceed the normal limit, directly interrupts the operation of power system. Moreover, the frequency oscillations may experience serious stability problems usually in the form of low frequency oscillations due to insufficient system damping. To overcome this problem, this paper not only takes the advantage of power modulation control offered by HVDC link to enhance the system damping, but also extends to stabilize frequency oscillations in an AC power system. By utilizing the interconnections between AC power systems as control channels of power modulation of HVDC link, this creates a new application of the HVDC link to stabilize frequency oscillations.

Due to unnecessary error in the past, conventional PI controller does not provide adequate control performance. The difficulty in obtaining the optimum settling time of previously said controller is mitigated by using FLC [7-9], which gives the opportunity to describe the control action in qualitative term and symbolic form. The proposed control can also be coordinated with conventional integral control for greater efficiency.

\section{PROBLEM FORMULATION OF PROPOSED CONTROL}

A typical two area interconnected system through AC -DC links is shown in Fig.1. At area -1 there is a DC transmission line and an inverter, whereas in area -2 , the HVDC link mainly consists of a rectifier. Originally area 2 supplied power PAC through AC line only, to area 1 . It is assumed that in area 1 there are large loads changing suddenly, like magnetic levitation transportation, huge steel mills, are furnaces etc. These cause in area 1, sudden demand for electric power and serious problem of frequency oscillations. In area 1, in addition, there are many independent power producers, having no frequency control abilities sufficiently. As the capabilities of frequency control governors in area 1 are not sufficient, they are not capable of stabilizing the frequencies oscillations. Whereas area 2 has sufficient frequency 
control capability to compensate for area-1. AC line alone cannot supply the required bulk power. To supply move power to area-1, an AC tie-line in parallel with an HVDC link is also provided from area 2. Through HVDC link, area-2 offers frequency stability to area-1[4]. The proposed method has large capability of frequency stabilisation to other interconnected areas having less capability. The proposed control can serve as a new auxiliary service for stabilising future

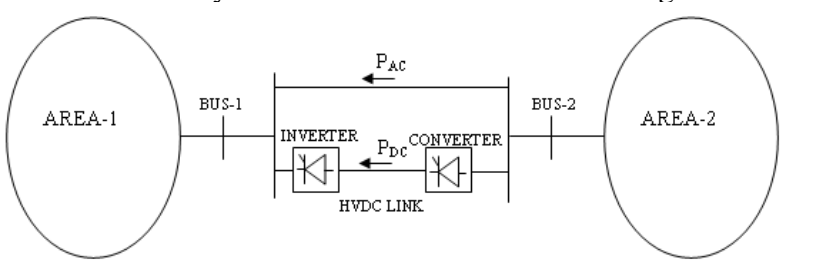

Fif. 1. An HVDC Link in a parallel AC-DC interconnected power system

nregulated power systems.

\section{DESIGN OF POWER MODULATION CONTROLLER BY HVDC LINK}

\section{Coordinated Control of HVDC Link and Governors}

To simplify the control design of the power modulation controller, the concept of coordinated control of HVDC link and governors will be explained. The HVDC link is superior to the governor which is a conventional frequency control system in terms of high-speed performance. Based on this different speed performance, a coordinated control of HVDC link and governors is as follows. When some sudden load disturbances occur in an area, an HVDC link quickly starts the control system to suppress the peak value of transient frequency deviation. Subsequently, governors eliminate the steady state error of the frequency deviation. Another advantage in considering the different speed performance is that the dynamics of governors in both areas can be neglected in the control design of HVDC link for simplicity.

\section{Control Design}

The Linearized Model of Interconnected Power System without Governors for Control Design of Power Modulation Controller of HVDC Link is delineated in Fig. 2 where the dynamics of governors in both areas are eliminated. The power modulation controller is modeled as a proportional controller of active power. It should be noted that the power modulation output of HVDC link ( $\triangle \mathrm{PDC})$, acting positively on an area, reacts negatively on another area in an interconnected system. $\triangle \mathrm{PDC}$, therefore, flows into both areas with different sign $(+,-)$, simultaneously. Here, to simplify the control design, the state equation of the system in Fig 2 where the time constant TDC is ignored, can be expressed as

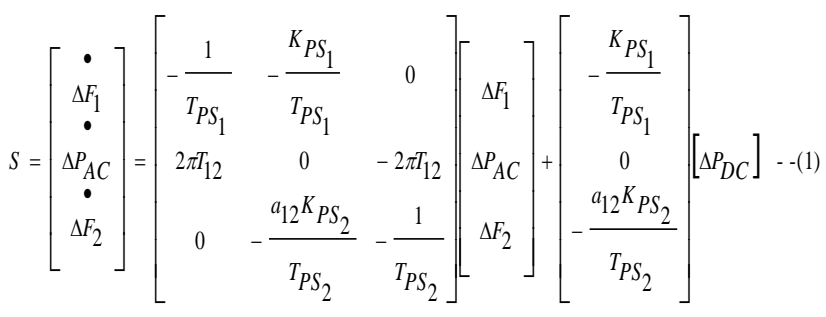

Equation (1) is referred to as system " $\mathrm{S}$ ". The variables and parameters of in Fig 2 are defined as follows. $\Delta \mathrm{f} 1, \Delta \mathrm{f} 2$ are frequency deviations of areas 1 and 2 respectively. $\triangle \mathrm{PAC}$ is an $\mathrm{AC}$ tie line power deviation between areas 1 and 2. $\triangle \mathrm{PDC}$ is a power modulation by HVDC link. $\triangle \mathrm{P} 12$ is the total tie line power deviations $(\triangle \mathrm{PAC}+\triangle \mathrm{PDC})$. Let $\mathrm{M} 1, \mathrm{M} 2$ be the inertia constants of areas 1 and 2. D1, D2 be the damping coefficients of areas 1 and 2 . A12 be an area capacity ratio between areas 1 and 2 .

Here, the control scheme for power modulation of HVDC link $(\triangle \mathrm{PDC})$ is designed by the eigenvalue assignment method, so that the dynamic aspect of the inter-area oscillation mode between areas 1 and 2 is specified. This mode can be explicitly expressed after applying the variable transformation.

$\mathrm{Y}=\mathrm{CX}$ $---(2)$

Where, $\mathrm{C}$ is a transformation matrix, $\mathrm{Y}$ is the transformed state vector, and $\mathrm{X}$ is the state vector in (1). Therefore, the transformed system can be expressed as

$\left[\begin{array}{c}\dot{\Delta Y_{1}} \\ \dot{\Delta Y_{2}} \\ \dot{\Delta}_{3}\end{array}\right]=\left[\begin{array}{ccc}\alpha & \beta & 0 \\ -\beta & \alpha & 0 \\ 0 & 0 & \lambda\end{array}\right]\left[\begin{array}{c}\Delta Y_{1} \\ \Delta Y_{2} \\ \Delta Y_{3}\end{array}\right]+\left[\begin{array}{c}\gamma_{1} \\ \gamma_{2} \\ 0\end{array}\right]\left[\Delta P_{D C}\right]$

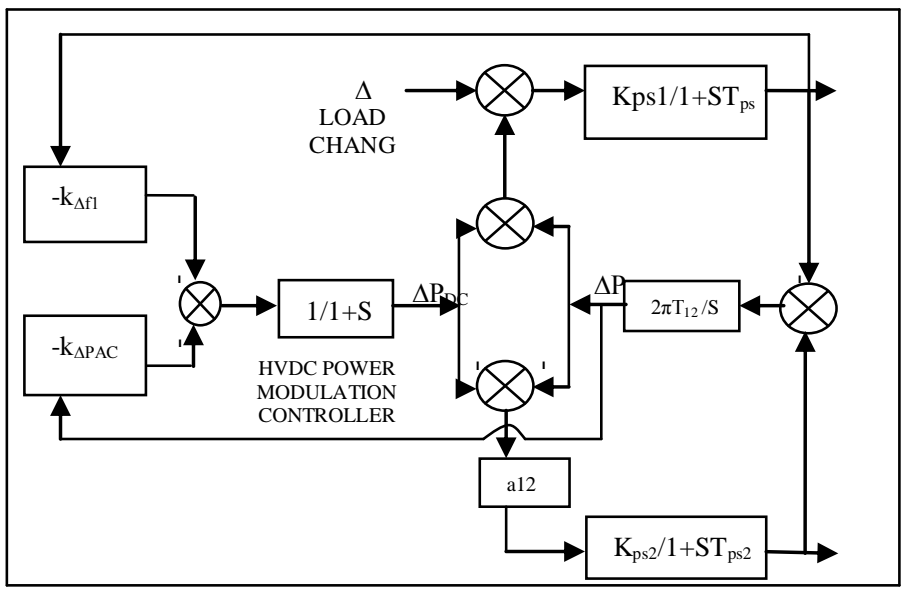

Fig. 2. Linearized Model of Interconnected Power Svstem without Governors for Control Design of 
The transformed coefficient matrix of equation (3) consists of two diagonal blocks with complex eigenvalue $\alpha \pm j \beta$ and real eigenvalue $\lambda$. The complex eigenvalue physically correspond to the inter-area oscillation mode, while the real eigenvalue represents the system inertia center mode. From the physical view point, it should be noticed that the HVDC link between two areas is effective to stabilize the inter area mode only, and therefore the input term of (3) corresponding to $\Delta y 3$ is zero. This means that the HVDC link cannot control the inertia center mode. To solve this crux, it is expected that the governors in both areas are responsible for suppressing the frequency deviation due to the inertia mode. Therefore, the power modulation controller of HVDC link is designed based on stabilizing the inter-area mode.

In order to extract the subsystem where the inter area oscillation mode between areas 1 and 2 is preserved, from the system $S$, the technique of overlapping decompositions 15 is applied. First, the state variables of the original system $S$ are classified into three groups, ie $x 1=[\Delta f 1], x 2=[\triangle P A C]$ and $x 3=$ $[\Delta f 2]$. According to the process of overlapping decompositions $^{[4]}$, the system $S$ can be expanded as

$$
\tilde{S}=\left[\begin{array}{c}
\bullet \\
Z_{1} \\
\bullet \\
Z_{2}
\end{array}\right]=\left[\begin{array}{cccc}
A_{11} & A_{12} & 0 & A_{13} \\
A_{21} & A_{22} & 0 & A_{23} \\
A_{21} & 0 & A_{22} & A_{23} \\
A_{31} & 0 & A_{32} & A_{33}
\end{array}\right]\left[\begin{array}{c}
Z_{1} \\
Z_{2}
\end{array}\right]+\left[\begin{array}{c}
B_{11} \\
B_{21} \\
B_{21} \\
B_{31}
\end{array}\right]\left[\Delta P_{D C}\right] \ldots \text {. . (4) }
$$

Where

$$
Z_{1}=\left[x_{1}^{T}, x_{1}^{T}\right]^{\mathrm{T}} \text { and } Z_{1}=\left[x_{1}^{T}, x_{1}^{T}\right]^{\mathrm{T}} \text {. The }
$$

element $a_{i j}, b_{i 1}(i, j=1,2,3)$ correspond to each element in the coefficient matrix in (1). The system $S$ in (4) can be decomposed into two interconnected overlapping subsystems,

$$
\begin{aligned}
& \tilde{S}_{1}=\dot{Z}_{1}=\left(\left[\begin{array}{ll}
A_{11} & A_{12} \\
A_{21} & A_{22}
\end{array}\right]\left[Z_{1}\right]+\left[\begin{array}{l}
B_{11} \\
B_{21}
\end{array}\right]\left[\Delta P_{D C}\right]\right)+\left[\begin{array}{ll}
0 & A_{13} \\
0 & A_{23}
\end{array}\right]\left[Z_{2}\right] \cdots(5) \\
& \tilde{S}_{2}=\dot{Z}_{2}=\left(\left[\begin{array}{ll}
A_{22} & A_{23} \\
A_{32} & A_{33}
\end{array}\right]\left[Z_{2}\right]\right)+\left(\left[\begin{array}{l}
B_{21} \\
B_{31}
\end{array}\right]\left[\Delta P_{D C}\right]\right)+\left[\begin{array}{ll}
A_{21} & 0 \\
A_{31} & 0
\end{array}\right]\left[Z_{1}\right] \ldots(6)
\end{aligned}
$$

The state variable $\mathrm{x} 2$, ie the $\mathrm{AC}$ tie line power deviation $(\triangle \mathrm{PDC})$ between both areas, is repeatedly included in both subsystems, which implies "Overlapping Decompositions".

For system stabilization, consider two interconnected subsystems S 1and S 2. The terms in the right hand sides of (5) and (6) can be separated into the decoupled subsystems (as indicated in the parenthesis in (5) and (6)) and the interconnected subsystems. As mentioned in Ikeda et al15, if each decoupled subsystem can be stabilized by its own input, the asymptotic stability of the interconnected overlapping subsystems S 1 and S 2 are maintained. Moreover, the asymptotic stability of the original system $\mathrm{S}$ is also guaranteed.
Consequently, the interactions with the interconnected subsystems in (5) and (6) are regarded as perturbations and are neglected during control design. As a result, the decoupled subsystems of $\mathrm{S} 1$ and $\mathrm{S} 2$ can be expressed as

$$
\begin{aligned}
& \tilde{S}_{D 1}=Z 1=\left[\begin{array}{ll}
a_{11} & a_{12} \\
a_{21} & a_{22}
\end{array}\right] Z 1+\left[\begin{array}{l}
b_{11} \\
b_{21}
\end{array}\right] \Delta P_{D C}-----(7) \\
& \tilde{S}_{D 2}=Z 2=\left[\begin{array}{ll}
a_{22} & a_{23} \\
a_{32} & a_{33}
\end{array}\right] Z 2
\end{aligned}
$$

In (7) and (8), there is a control input $\triangle \mathrm{PDC}$ appearing only in subsystem S D1 . Here, the decoupled subsystem S D1 is regarded as the designed system, which can be expressed as $\left[\begin{array}{c}\dot{\Delta}_{1} \\ \Delta P_{A C}\end{array}\right]=\left[\begin{array}{cc}-\frac{1}{T_{P S_{1}}} & -\frac{K_{P S_{1}}}{T_{P S_{1}}} \\ 2 \pi T_{12} & 0\end{array}\right]\left[\begin{array}{c}\Delta F_{1} \\ \Delta P_{A C}\end{array}\right]+\left[\begin{array}{c}-\frac{K_{P S_{1}}}{T_{P S_{1}}} \\ 0\end{array}\right]\left[\Delta P_{D C}\right]-----(9)$

It can be verified that the eigenvalues of (9) are $\alpha \pm j \beta$, ie, the inter-area oscillation mode in the system $\mathrm{S}$. It should be noticed that by virtue of overlapping decompositions, the physical characteristic of the original system is still preserved after the process. Here, the control purpose of HVDC link is to damp the peak value of frequency deviation after sudden load disturbance. By eigenvalue assignment method, the feedback control scheme of $\triangle \mathrm{PDC}$ can be expressed as

$$
\Delta P_{D C}=-k_{\Delta f 1} \Delta f_{1}-k_{\Delta P_{A C}} \Delta P_{A C}-----(10)
$$

Note that, the state feedback scheme is constructed by two measurable signals, ie a frequency deviation of area 1 and an AC tie-line power deviation. The integral controller has received a great deal of attention in the process control areas. Integral controller is a device that produces an output signal, which is integral to the input signal. The system control outputs, which depends on the area control error, that is given by

$$
\begin{aligned}
& U_{1}=K_{I_{1}} \int A C E_{1}(t) d t \\
& U_{2}=K_{I_{2}} \int A C E_{2}(t) d t
\end{aligned}
$$

Where $A C E_{1}=\beta_{1} \Delta F_{1}+\Delta P_{+i e 1}$

$$
\begin{aligned}
& A C E_{2}=\beta_{2} \Delta F_{2}+\Delta P_{+i e 2} \\
\Delta P_{+i e 2}= & a_{12} \Delta P_{+i e 1}
\end{aligned}
$$

$\beta$ is the biasing factor and $\mathrm{U} 1 \& \mathrm{U} 2$ is the system control output. The integral controller is successful approach towards the zero steady state error in the frequency of the system. The conventional integral controller, the gain $\mathrm{KI}$ has been determined using Integral Square Error (ISE) criterion. The objective function used for this technique is 


$$
J=\int_{0}^{t}\left(\Delta F_{1}^{2}+\Delta F_{2}^{2}+\Delta P_{+i e}^{2}\right) d t
$$

Because of the complexity and multivariable condition of the power system, a conventional model may not give satisfactory solution. This inference is further reinforced by fuzzy logic controller which is useful in mitigating a wide range of control problem.

\section{Fuzzy Logic Controller:}

Based on the earlier research results of this stabilisation of frequency in the interconnected power system ${ }^{[10-12]}$, we can design the fuzzy logic controller. Fuzzy set theory and fuzzy logic establish the rule of a nonlinear mapping. The use of fuzzy sets provides a basic for a systematic ways for the application of uncertain and indefinite models. A two area interconnected power system with parallel AC - DC link is considered here as shown in fig. 3 .

The overall system can be modelled as a multivariable system in the form of

$$
\mathrm{X}=\mathrm{Ax}+\mathrm{Bu}+\mathrm{Ed}
$$

Where the System State vector $\mathbf{x}$ consists of nine variables as

$$
\begin{aligned}
& \text { System control input is }[\mathrm{u}]=\left|\begin{array}{l}
\mathrm{u}_{1} \\
\mathrm{u}_{2}
\end{array}\right|=\left|\begin{array}{c}
\Delta \mathrm{P}_{\mathrm{c}} \\
\Delta \mathrm{P}_{\mathrm{c} 2}
\end{array}\right| \\
& \text { System disturbance input vector is }[\mathrm{d}]=\left|\begin{array}{l}
\mathrm{d}_{1} \\
\mathrm{~d}_{2}
\end{array}\right|=\left|\begin{array}{c}
\Delta \mathrm{P}_{\mathrm{d}} \\
\Delta \mathrm{P}_{\mathrm{d} 2}
\end{array}\right|
\end{aligned}
$$

$\mathrm{A}, \mathrm{B}$ and $\mathrm{E}$ are system state matrix, distribution matrix and disturbance distribution matrix of appropriate dimensions.

The main objective is to minimize the mean square error (MSE) of the area control error (ACE) due to step type load disturbance ${ }^{[13-15]}$. The objective function of the load frequency controller [4] is given by,

$$
\mathrm{ACE}_{\mathrm{i}}=\beta \Delta \mathrm{F}_{\mathrm{i}}+\Delta \mathrm{P}_{\text {tiei }}
$$

Where $\mathrm{i}$ - vary from 1 to 2

$\beta$ is the biasing factor.
The design of LFC can be normally divided into three areas namely allocation of area inputs, determination of rules and defuzzifying of output into a real value.

The method of fuzzification has found increasing application in power system. In this FLC, membership function (MF) specifies the degree to which a given input belongs to a set. In the case of FLC, seven membership functions in triangular shape have been chosen for the inputs of $\Delta \mathrm{F}_{\mathrm{i}}, \Delta \mathrm{P}_{\text {tiei }}$ and output $(\mathrm{u})$. The linguistic descriptions of input membership functions are Negative Large (NL), Negative Medium (NM), Negative Small (NS), Zero (ZE), Positive Small (PS), Positive Medium (PM) and Positive Large (PL). The output membership functions are Negative High (NH), Negative Medium (NM), Negative Small (NS), Zero (ZE), Positive Small (PS), Positive Medium (PM) and Positive High (PH).the fuzzy membership functions for inputs and output are shown in Figs. 4 and 5. The union maximum operation has been selected for the fuzzy implication. For the two- input fuzzy system, it is generally expressed as

$$
\mu_{\mathrm{Ai}(\mathrm{x} 1) \mathrm{U} \text { Ai( } \mathrm{x} 2)}=\max \left\{\mu_{\mathrm{Ai}(\mathrm{x} 1)}, \mu_{\mathrm{Ai}(\mathrm{x} 2)}\right\}
$$

where $A_{i(x 1)}$ and $A_{i(x 2)}$ are input fuzzy sets. The rule base of the fuzzy controller relates the premise $\left(\Delta\right.$ Fand $\left.\Delta \mathrm{P}_{\text {tie }}\right)$ to consequent $(\mathrm{u})$. The structure of the control rules of the fuzzy controller with two inputs and an one output is expressed as
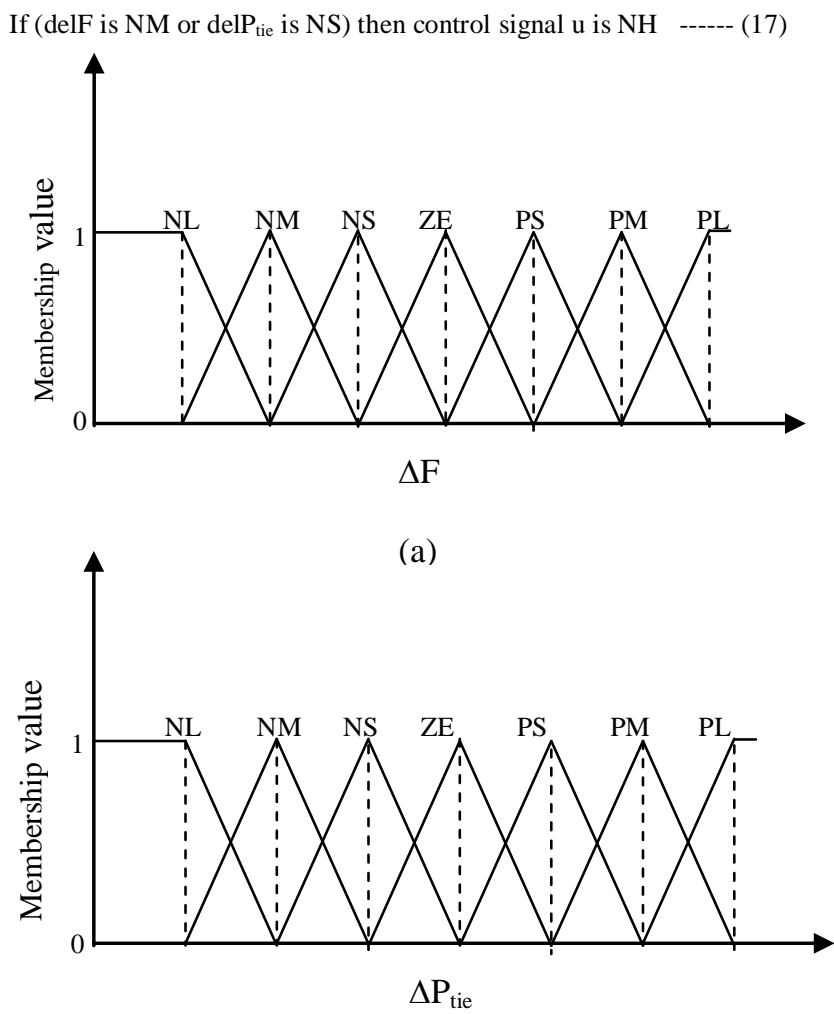

(b)

Fig.5.Input membership functions of fuzzy controller (a) $\Delta \mathrm{F}$; (b) $\Delta \mathrm{P}_{\text {tie }}$ 


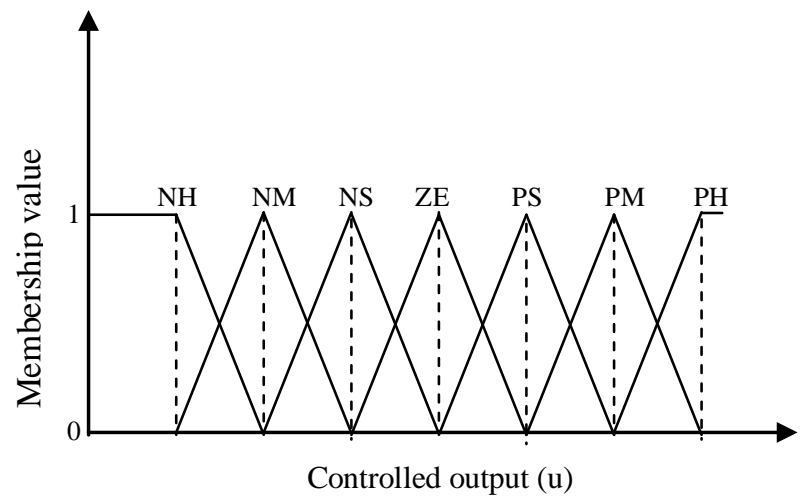

Fig.6 Output membership functions of fuzzy controller

Table 1 lists 49 linguistic fuzzy rules for the fuzzy controller. The centroid difuzzification has been made to find the crisp value of output. The centroid difuzzification is defined as

$$
\mathrm{U}^{\text {Crisp }}=\frac{\sum_{\mathrm{i}=1} \mathrm{u}_{\mathrm{i}} \mu\left(\mathrm{u}_{\mathrm{i}}\right)}{\sum_{\mathrm{i}=1} \mu\left(\mathrm{u}_{\mathrm{i}}\right)}
$$

Table - 1, Fuzzy Control rules

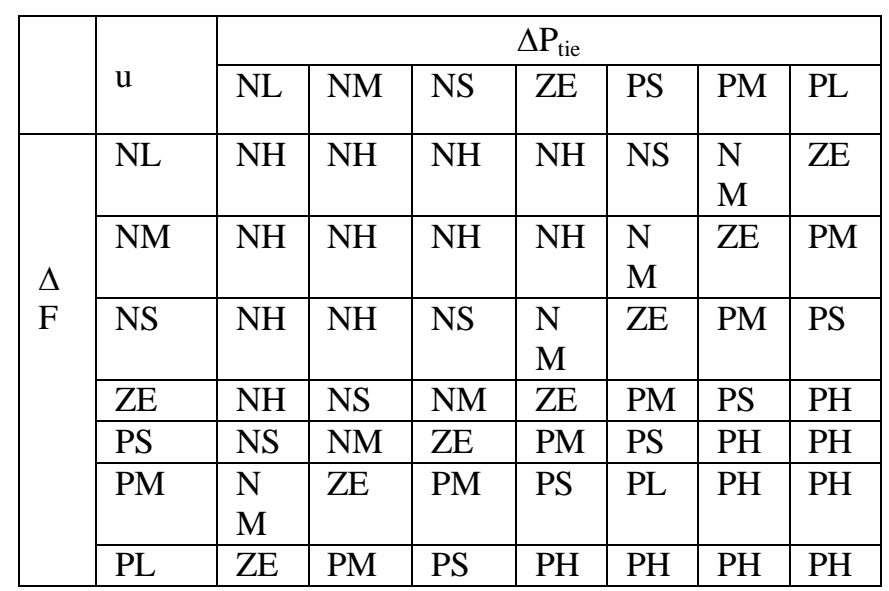

Where $\mathrm{U}^{\text {Crisp }}$ is the output ofthe fuzzy controller, $\mathrm{u}_{\mathrm{i}}$ denotes the centre of the membership function of the consequent of the $i$-th rule, $\mu$ denotes the membership value for the rule's premise and $\mathrm{n}$ reperents the total number of fuzzy rules.

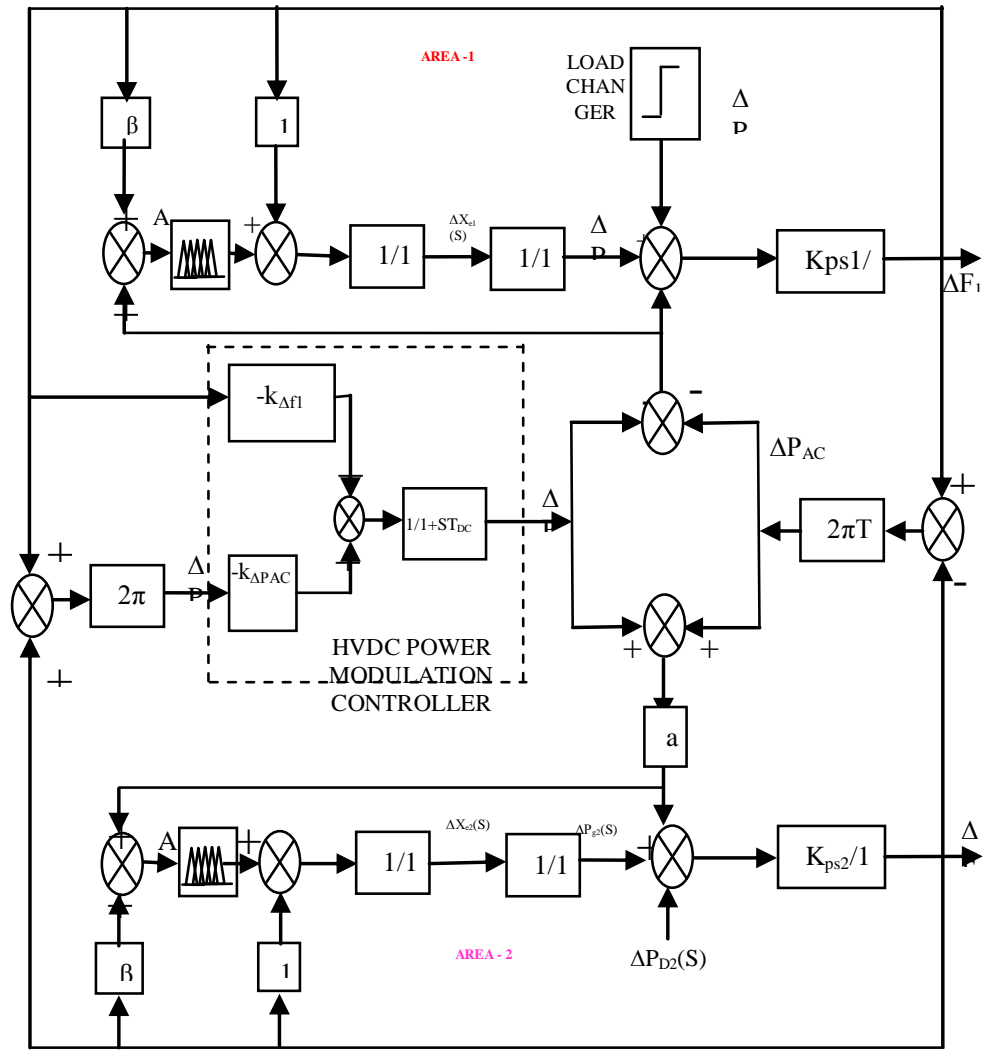

Fig. 3. Modeling of HVDC power modulator in the two area thermal non reheat with parallel AC-DC power system

\section{Simulation Result and Discussion:}

In this paper, a two area non reheat interconnected system is used to design and evaluate the effects of the power modulation controller of HVDC link. The system data are given in the appendix. The simulation study is carried out by the software "Mat lab".

The system is simulated for a step- load such as a large steel roll mill and arc- furnace factory, an increase $10 \%$ (0.1p.u.M.W.) occurs in area- 1 . Due to this, change in dynamic responses of the system has been observed. Fig.

4 and 5 indicates the frequency deviation of area $1 \& 2$ and fig. 6 indicates the tie line power deviation for a step load disturbance of area- 1 .

Similarly the system is simulated for a step- load disturbance of $30 \%$ (0.3 p.u.M.W.) occurs in area- 1.For this disturbance, the frequency deviation of area $1 \& 2$ is shown in fig. $7 \& 8$. The tie line power deviation for a step load disturbance of area- 1 is shown in Fig.9. The effectiveness of the proposed control method is demonstrated by comparing the integral of the square of the error (ISE), integral of the absolute value of the error (IAE) and settling time. 
The ISE and IAE are given as

$$
\begin{aligned}
& I S E=\int_{0}^{\infty} A C E^{2} d t \\
& I A E=\int_{0}^{\infty}|A C E| d t
\end{aligned}
$$

The performance comparison for the step load disturbance of $10 \%$ and $30 \%$ are presented in Table- 2

Table-2, Performance Comparison of different Control Algorithms

\begin{tabular}{|c|c|c|c|c|c|c|}
\hline \multirow{2}{*}{$\begin{array}{c}\text { Types of } \\
\text { Control }\end{array}$} & \multicolumn{2}{|c|}{ ISE } & \multicolumn{2}{c|}{ IAE } & \multicolumn{2}{c|}{ Settling Time $\mathrm{T}_{\mathrm{s}}(\mathrm{S})$} \\
\cline { 2 - 7 } & $\begin{array}{c}10 \% \\
\text { Disturbance }\end{array}$ & $\begin{array}{c}30 \% \\
\text { Disturbance }\end{array}$ & $\begin{array}{c}10 \% \\
\text { Disturbance }\end{array}$ & $\begin{array}{c}30 \% \\
\text { Disturbance }\end{array}$ & $\begin{array}{c}10 \% \\
\text { Disturbance }\end{array}$ & $\begin{array}{c}30 \% \\
\text { Disturbance }\end{array}$ \\
\hline $\begin{array}{c}\text { Integral } \\
\text { Control }\end{array}$ & 0.0156 & 0.141 & 0.24 & 0.75 & 16.2 & 18.6 \\
\hline $\begin{array}{c}\text { Fuzzy } \\
\text { Control }\end{array}$ & 0.00138 & 0.011 & 0.056 & 0.168 & 9.4 & 11.4 \\
\hline
\end{tabular}

It is observed that the fuzzy logic controller exhibits relatively good performances and fast settling time. The conventional integral controller does not yield adequate control performance.. Simulation result concludes that fuzzy logic controller yields much improved control performance than the conventional integral controller.

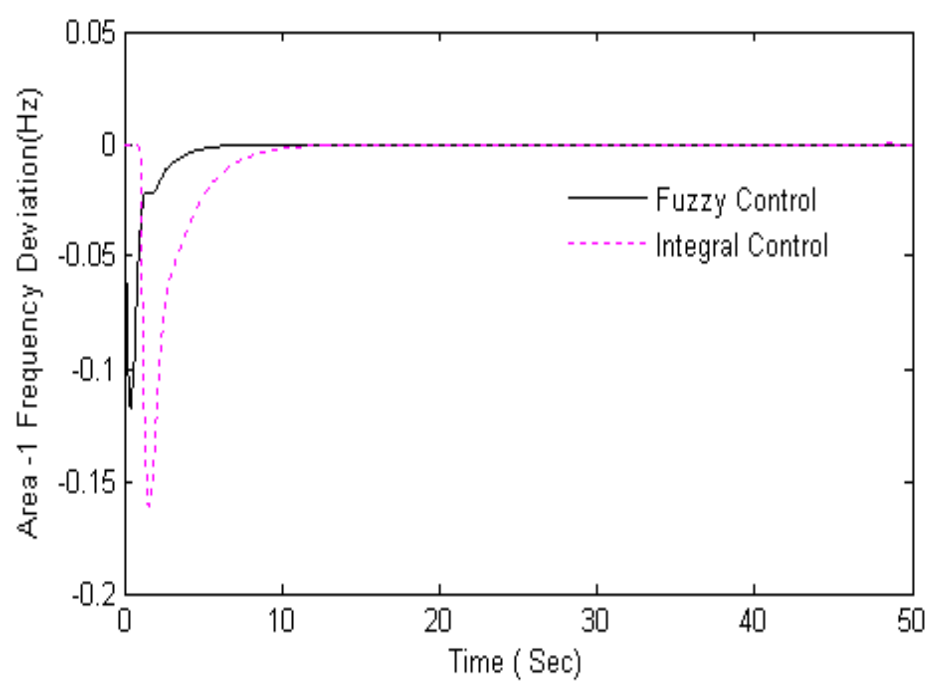

Fig.4. Frequency deviation in area-1(10\% Disturbance)

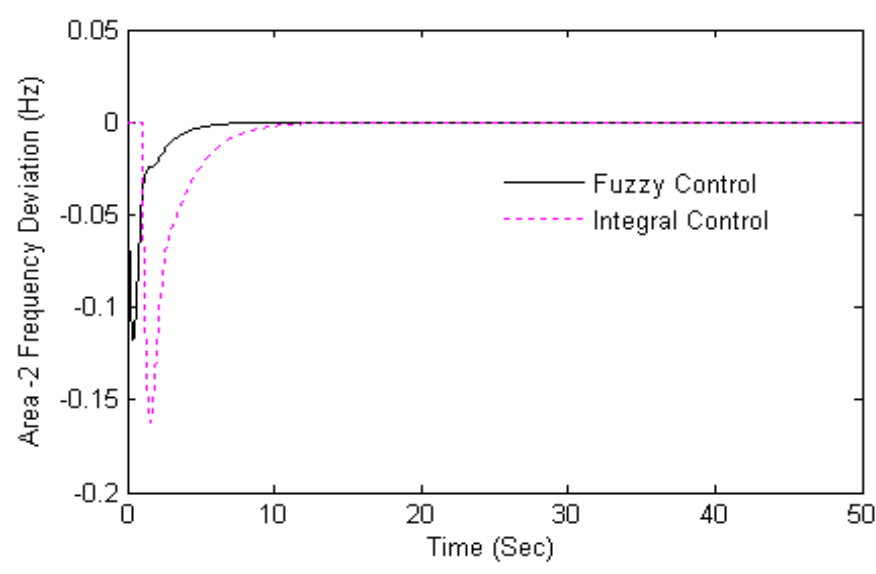

Fig.5. Frequency deviation in area-2(10\% Disturbance)

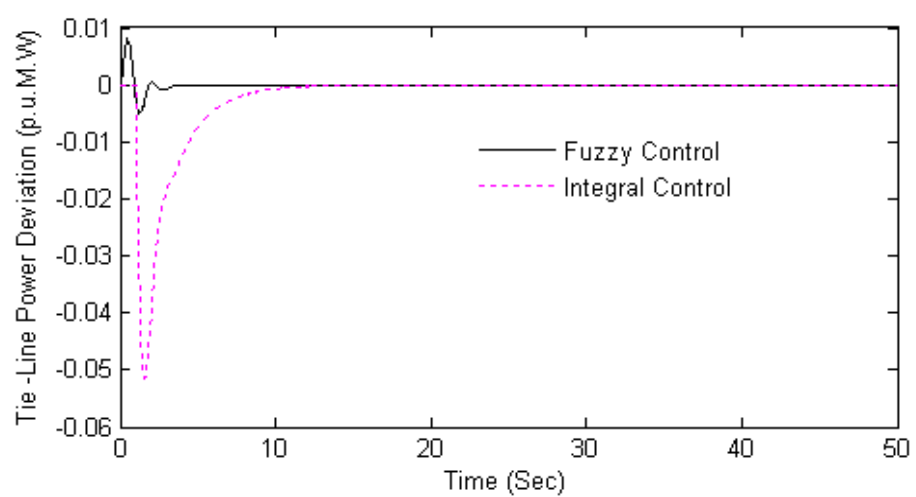

Fig.6. Tie line power deviation (10\% Disturbance)

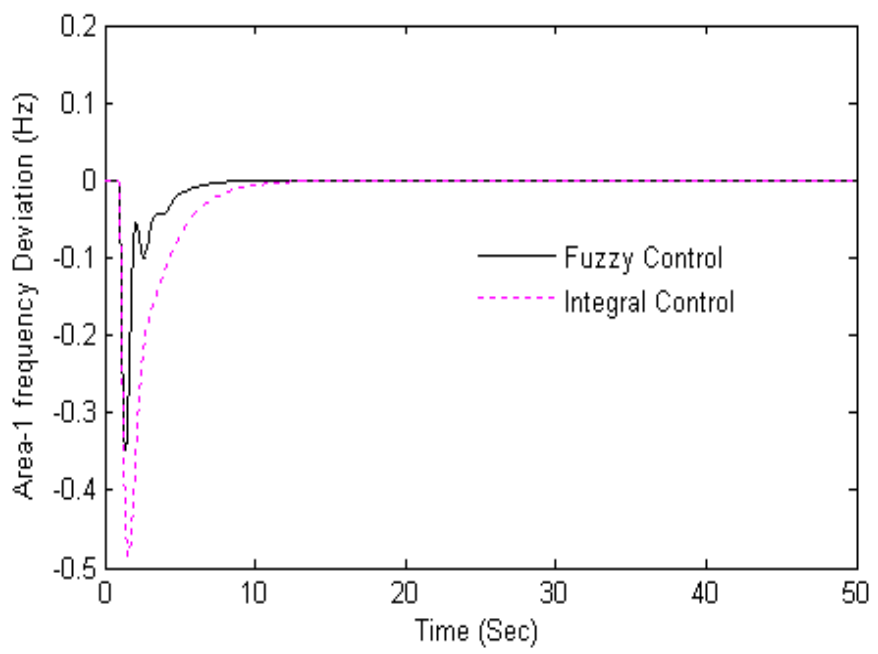

Fig.7. Frequency deviation in area-1(30\% Disturbance) 


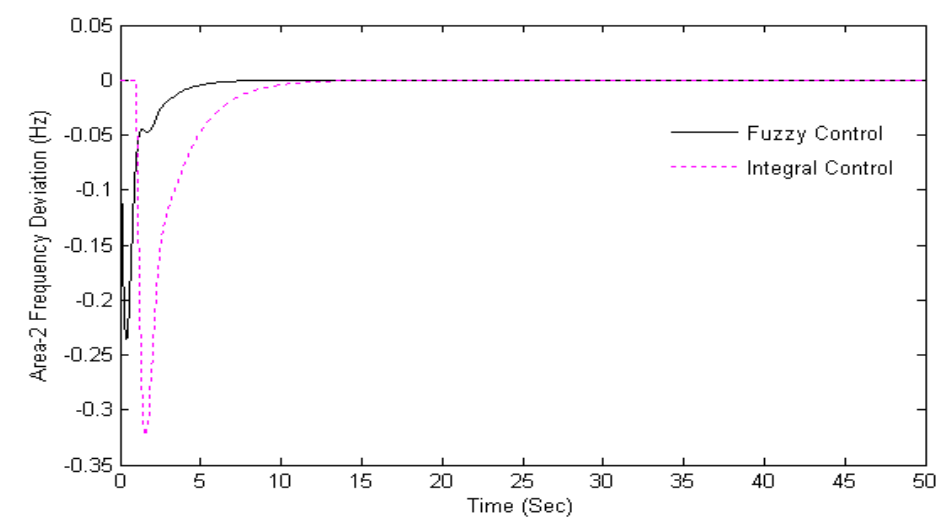

Fig.8. Frequency deviation in area-2(30\% Disturbance)

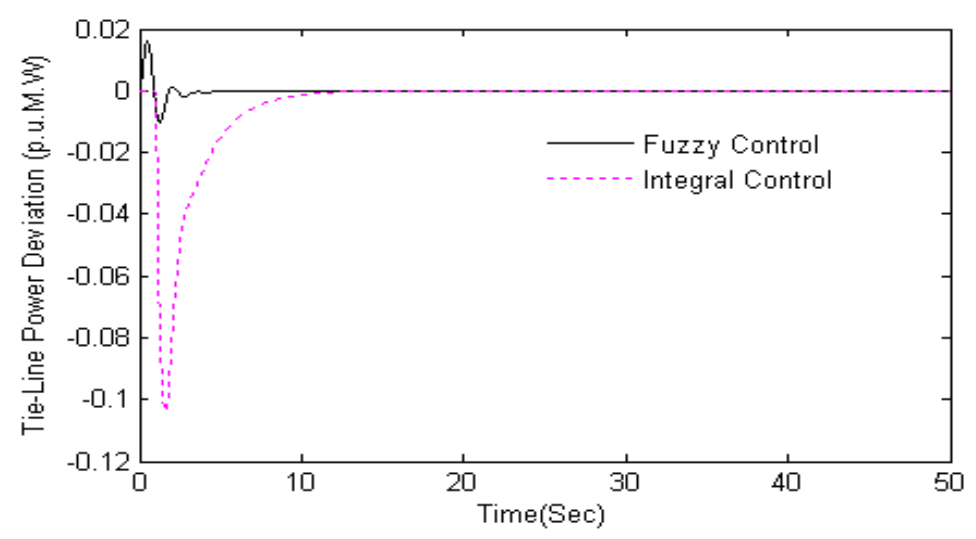

Fig.9. Tie line power deviation (30\% Disturbance)

\section{Conclusion:}

In this paper, a sophisticated method for stabilizing frequency oscillations in a parallel AC- DC interconnected power system through an HVDC link to stabilize frequency oscillations in AC power system can be achieved. The HVDC power modulation controller is developed by applying decomposition and eigenvalue assignment techniques. By simulation study, the fuzzy logic controller is very effective in suppressing the frequency oscillations caused by rapid load disturbances. For further study, the proposed control design of HVDC link will be extended to stabilize the frequency oscillations in a multi area interconnected power system.

\section{Acknowledgements:}

We would like to thank Prof. K. Nallathambi and Prof. N. Kanagaraj, Department of Electrical and Electronics Engineering, K.S.R. College of Engineering, Tiruchengode, Tamilnadu, India, for their valuable suggestions throughout this work. We also thank our management trustee K.S.R. College of Engineering for the facilities provided to prepare this paper.

\section{REFERENCES:}

[1] George Gross and Jeong Woo Lee, "Analysis of Load Frequency Control Performance Assessment Criteria",IEEE Transaction on Power Systems", vol 16,No. 3, August 2001 , pp520- 525 .

[2] Dulpichet Rerkpreedapong, Amer Hasanovic and Ali Feliachi, " Robust Load Frequency Control using Genetic Algorithms and Linear Matrix Inequalities", IEEE Transaction on Power Systems, vol 18,No. 2, May 2003 , pp855-861.

[3]F.P.deMellow, R.J.Mills and W.F.B'Rells, “Automatic Generation control”, Part II- Digital control Techniques, IEEE Transaction on Power Apparatus and Systems", vol. PAS-92, No.23, May 1972 , pp716- 724.

[4] Issarachai Ngamroo, "A stabilization of frequency oscillations in a parallel AC - DC interconnecte power systems via an HVDC link", scienceAsia28 (2002), pp 173180.

[5] Chidambaram and A,Velusamy.S., "Decentralized Biased Controllers for Load Frequency Control of Interconnected Power Systems Considering Governor Dead band NonLinearity”, IEEE Indicon, Dec 2005, pp521-525.

[6] Masiala.M,Ghribi.M and Kaddouri.A,“An Adaptive Fuzzy Controller Gain Scheduling for Power System LoadFrequency Control”, IEEE (ICIT), 2004 , pp 1515-1520.

[7] J. Talaq and F. Al-Basri, "Adaptive Fuzzy Gain Scheduling for Load Frequency Control", IEEE Transaction on Power Systems", vol 14, No. 1, February 1999, pp145- 150.

[8] Vaibhav Donde, M.A. Pai and Ian A. Hiskens, " Simulation and Optimization in an AGC System after

Deregulation" IEEE Transaction on Power Systems, vol 16,No. 3, August 2001 , pp481- 487.

[9] Anand.B and Ebenezer Jeyakumar.A., “ Load Frequency Control with Fuzzy Logic Controller Considering Non -

Linearities and Boiler Dynamics"ACSE, vol8, issue 3, January2009, pp $15-20$.

[10] Mathur.H.D. and Manjunath.H.V., “ Frequency Stabilisation using Fuzzy Logigbased controller for multiarea

power system", South Pacific journal of National Science, vol.4, 2007, pp 22- 30.

[11] Chia- Feng Juang and Chun-Feng Lu, "Power System Load Frequency Control By Genetic Fuzzy gain scheduling

Controller", Journal of the Chinese institute of Engineers,vol.28,No.6,2005, pp 1013 - 1018. 
[12] Chia- Feng Juang and Chun-Feng Lu, "Power System Load Frequency Control By Evolutionary Fuzzy PI Controller", IEEE, July2004, pp715-719.

[13] Chowdhury. S, Chaudhury. S.P and Choudhuri.S, "Advanced digital load frequency control with unknown deterministic power demand for interconnected power systems", IE (I) journal- EL vol.80, Nov 1999, pp 87- 95.

[14] Dulpichet Rerkpreedapong and Ali Feliachi, "PI Gain Scheduler for Load Frequency Control Using Spline techniques",IEEE,2003, pp259-263.

[15] Ibraheem, A.Kumar and D.P.Kothari," recent Philosophies of Automatic Generation Control Strategies in Power Systems", IEEE Transaction on Power Systems, vol 20,No. 1, February 2005, pp340- 357.

\section{Appendix:}

Two area Non - reheat thermal power system

$$
\begin{array}{ll}
\beta_{1} & =\beta_{2}=0.425 \text { p.u.M..W } / \mathrm{Hz} . \\
\mathrm{T}_{\mathrm{t} 1} & =\mathrm{T}_{\mathrm{t} 2}=0.3 \mathrm{sec} \\
\mathrm{T}_{\mathrm{g} 1} & =\mathrm{T}_{\mathrm{g} 2}=0.08 \mathrm{sec} \\
\mathrm{K}_{\mathrm{ps} 1} & =\mathrm{K}_{\mathrm{ps} 2}=120 \mathrm{~Hz} / \text { p.u. MW } \\
2 \pi \mathrm{T}_{12} & =0.545 \\
\mathrm{~T}_{\mathrm{ps} 1} & =\mathrm{T}_{\mathrm{ps} 2}=20 \mathrm{sec} \\
\mathrm{R}_{1} & =\mathrm{R}_{2}=2.4 \mathrm{~Hz} / \mathrm{p} . \mathrm{u} \mathrm{MW} . \\
\mathrm{K}_{\Delta \mathrm{f} 1}=-0.3201 & \\
\mathrm{~K}_{\Delta \mathrm{PAC}}=-2.144
\end{array}
$$

